\title{
Intertwinors on Functions over the Product of Spheres
}

Doojin HONG

Department of Mathematics, University of North Dakota, Grand Forks ND 58202, USA

E-mail: doojin.hong@und.edu

Received August 23, 2010, in final form December 30, 2010; Published online January 06, 2011

doi:10.3842/SIGMA.2011.003

\begin{abstract}
We give explicit formulas for the intertwinors on the scalar functions over the product of spheres with the natural pseudo-Riemannian product metric using the spectrum generating technique. As a consequence, this provides another proof of the even order conformally invariant differential operator formulas obtained earlier by T. Branson and the present author.
\end{abstract}

Key words: intertwinors; conformally invariant operators

2010 Mathematics Subject Classification: 53A30; 53C50

\section{Introduction}

Branson, Ólafsson, and Ørsted presented in [8] a method of computing intertwining operators between principal series representations induced from maximal parabolic subgroups of semisimple Lie groups in the case where $K$-types occur with multiplicity at most one. One of the main ideas is that the intertwining relation, when "compressed" from a $K$-type to another $K$-type, can provide a purely numerical relation between eigenvalues on the $K$-types being considered through some relatively simple calculations. This procedure of getting a recursive numerical relation is referred to as "spectrum generating" technique.

When the group under consideration acts as conformal transformations, the intertwinors are conformally invariant operators (see [8, Section 3] for example) and they have been one of the major subjects in mathematics and physics.

Explicit formulas for such operators on many manifolds are potentially important. For instance, the precise form of Polyakov formulas in even dimensions for the quotient of functional determinants of operators only depends on some constants that appear in the spectral asymptotics of the operators in question [3].

In 1987, Branson [1] presented explicit formulas for invariant operators on functions and differential forms over the double cover $S^{1} \times S^{n-1}$ of the $n$-dimensional compactified Minkowski space. And Branson and Hong [6, 9, 10] gave explicit determinant quotient formulas for operators on spinors and twistors including the Dirac and Rarita-Schwinger operators over $S^{1} \times S^{n-1}$.

In this paper, we show that the spectrum generating technique can be applied to get explicit formulas for the intertwinors on the scalar functions over general product of spheres, $S^{p} \times S^{q}$ with the natural pseudo-Riemannian metric.

\section{Some background on conformally covariant operators}

We briefly review conformal covariance and the intertwining relation (for more details, see [4, 8]).

Let $(M, g)$ be an $n$-dimensional pseudo-Riemannian manifold. If $f$ is a (possibly local) diffeomorphism on $M$, we denote by $f$. the natural action of $f$ on tensor fields which acts on vector fields as $f \cdot X=(d f) X$ and on covariant tensors as $f \cdot \phi=\left(f^{-1}\right)^{*} \phi$. 
A vector field $T$ is said to be conformal with conformal factor $\omega \in C^{\infty}(M)$ if $\mathcal{L}_{T} g=2 \omega g$,

where $\mathcal{L}$ is the Lie derivative. The conformal vector fields form a Lie algebra $\mathfrak{c}(M, g)$. A conformal transformation on $(M, g)$ is a (possibly local) diffeomorphism $h$ for which $h \cdot g=\Omega^{2} g$ for some positive function $\Omega \in C^{\infty}(M)$. The global conformal transformations form a group $\mathscr{C}(M, g)$. We have representations defined by

$$
\begin{array}{ll}
\mathfrak{c}(M, g) \stackrel{U_{a}}{\longrightarrow} \text { End } C^{\infty}(M), & U_{a}(T)=\mathcal{L}_{T}+a \omega \quad \text { and } \\
\mathscr{C}(M, g) \stackrel{u_{a}}{\longrightarrow} \text { Aut } C^{\infty}(M), & u_{a}(h)=\Omega^{a} h .
\end{array}
$$

for $a \in \mathbb{C}$.

Note that if a conformal vector field $T$ integrates to a one-parameter group of global conformal transformation $\left\{h_{\varepsilon}\right\}$, then

$$
\left\{U_{a}(T) \phi\right\}(x)=\left.\frac{d}{d \varepsilon}\right|_{\varepsilon=0}\left\{u_{a}\left(h_{-\varepsilon}\right) \phi\right\}(x) .
$$

In this sense, $U_{a}$ is the infinitesimal representation corresponding to $u_{a}$.

A differential operator $D: C^{\infty}(M) \rightarrow C^{\infty}(M)$ is said to be infinitesimally conformally covariant of bidegree $(a, b)$ if

$$
D U_{a}(T) \phi=U_{b}(T) D \phi
$$

for all $T \in \mathfrak{c}(M, g)$ and $D$ is said to be conformally covariant of bidegree $(a, b)$ if

$$
D u_{a}(h) \phi=u_{b}(h) D \phi
$$

for all $h \in \mathscr{C}(M, g)$.

To relate conformal covariance to conformal invariance, we let $M$ be an $n$-dimensional manifold with metric $g$ and recall $[4,5]$ that conformal weight of a subbundle $V$ of some tensor bundle over $M$ is $r \in \mathbb{C}$ if and only if

$$
\bar{g}=\Omega^{2} g \Longrightarrow \overline{g_{V}}=\Omega^{-2 r} g_{V},
$$

where $\Omega>0 \in C^{\infty}(M)$ and $g_{V}$ is the induced bundle metric from the metric $g$. Tangent bundle, for instance, has conformal weight -1 . Let us denote a bundle $V$ with conformal weight $r$ by $V^{r}$. Then we can impose new conformal weight $s$ on $V^{r}$ by taking tensor product of it with the bundle $I^{(s-r) / n}$ of scalar $((s-r) / n)$-densities. Now if we view an operator of bidegree $(a, b)$ as an operator from the bundle with conformal weight $-a$ to the bundle with conformal weight $-b$, the operator becomes conformally invariant.

As an example, let us consider the conformal Laplacian on $M$ :

$$
Y=\triangle+\frac{n-2}{4(n-1)} R
$$

where $\triangle=-g^{a b} \nabla_{a} \nabla_{b}$ and $R$ is the scalar curvature. Note that $Y: C^{\infty}(M) \rightarrow C^{\infty}(M)$ is conformally covariant of bidegree $((n-2) / 2,(n+2) / 2)$. That is,

$$
\bar{Y}=\Omega^{-\frac{n+2}{2}} Y \mu\left(\Omega^{\frac{n-2}{2}}\right),
$$

where $\bar{Y}$ is $Y$ evaluated in $\bar{g}$ and $\mu\left(\Omega^{\frac{n-2}{2}}\right)$ is multiplication by $\Omega^{\frac{n-2}{2}}$. If we let $V=C^{\infty}(M)$ and view $Y$ as an operator

$$
Y: V^{-\frac{n-2}{2}} \rightarrow V^{-\frac{n+2}{2}},
$$

we have, for $\phi \in V^{-\frac{n-2}{2}}$,

$$
\bar{Y} \bar{\phi}=\overline{Y \phi},
$$

where $\bar{Y}, \bar{\phi}$, and $\overline{Y \phi}$ are $Y, \phi$, and $Y \phi$ computed in $\bar{g}$, respectively. 


\section{Conformal structure and intertwining relation}

Let us first briefly review conformal structure on $S^{p} \times S^{q}$ (for more details, see [12] for example).

Let $\mathbb{R}^{p+1, q+1}$ be the $(n+2)$-dimensional pseudo-Riemannian manifold $\mathbb{R}^{p+q+2}$ equipped with the pseudo-Riemannian metric

$$
-\xi_{-p}^{2}-\cdots-\xi_{0}^{2}+\xi_{1}^{2}+\cdots+\xi_{q+1}^{2}
$$

and define submanifolds of $\mathbb{R}^{p+1, q+1}$ by

$$
\begin{aligned}
& \Xi:=\left\{-\xi_{-p}^{2}-\cdots-\xi_{0}^{2}+\xi_{1}^{2}+\cdots+\xi_{q+1}^{2}=0\right\} \backslash\{0\}, \\
& M:=\left\{\xi_{-p}^{2}+\cdots+\xi_{0}^{2}=\xi_{1}^{2}+\cdots+\xi_{q+1}^{2}=1\right\} \simeq S^{p} \times S^{q} .
\end{aligned}
$$

The natural action of the multiplicative group $\mathbb{R}_{+}^{\times}=\{r \in \mathbb{R}: r>0\}$ on $\Xi$ identifies $\Xi / \mathbb{R}_{+}^{\times}$ with $M$ and the natural action of the orthogonal group $G:=O(p+1, q+1)$ on $\mathbb{R}^{p+1, q+1}$ stabilizes the metric cone $\Xi$ and these two actions commute. So we get a $G$-equivariant principal $\Xi / \mathbb{R}_{+}^{\times}$ bundle:

$$
\Phi: \Xi \rightarrow M, \quad\left(\xi_{-p}, \ldots, \xi_{0}, \xi_{1}, \ldots, \xi_{q+1}\right) \rightarrow \frac{1}{\sqrt{\xi_{-p}^{2}+\cdots+\xi_{0}^{2}}}\left(\xi_{-p}, \ldots, \xi_{0}, \xi_{1}, \ldots, \xi_{q+1}\right) .
$$

The standard pseudo-Riemannian metric on $\mathbb{R}^{p+1, q+1}$ induces the standard pseudo-Riemannian metric $-g_{S^{p}}+g_{S^{q}}$ on $S^{p} \times S^{q} \simeq M$. For $h \in G, z \in M$, the map

$$
z \stackrel{h}{\longrightarrow} h \cdot z \stackrel{\Phi}{\longrightarrow} \Phi(h \cdot z)
$$

is a conformal transformation on $M$ and $G$ is the full conformal group of $S^{p} \times S^{q}$.

A basis of the conformal vector fields on $S^{p} \times S^{q}$ in homogeneous coordinates is

$$
L_{\alpha \beta}=\varepsilon_{\alpha} \xi_{\alpha} \partial_{\beta}-\varepsilon_{\beta} \xi_{\beta} \partial_{\alpha},
$$

where $\partial_{\alpha}=\partial / \partial \xi_{\alpha}$ and $-\varepsilon_{p}=\cdots=-\varepsilon_{0}=\varepsilon_{1}=\cdots=\varepsilon_{q+1}=1$.

The subalgebra spanned by $L_{\alpha \beta}$ for $-p \leq \alpha, \beta \leq 0$ is a copy of $\mathfrak{s o}(p+1)$ so it generates $\mathrm{SO}(p+1)$ group of isometries. Likewise, the $L_{\alpha \beta}$ for $1 \leq \alpha, \beta=q+1$ generate $\mathrm{SO}(q+1)$ group of isometries.

Let $\mathrm{SO}_{0}(p+1, q+1)$ be the identity component of $G$ and note $K=\mathrm{SO}(p+1) \times \mathrm{SO}(q+1)$ is a maximal compact subgroup of $\mathrm{SO}_{0}(p+1, q+1)$. Elements in $K$ act as isometries on $S^{p} \times S^{q}$ and proper conformal vector fields are the ones with mixed indices: $L_{\alpha \beta}$ for $-p \leq \alpha \leq 0<\beta \leq q$.

To express the typical proper conformal vector field $L_{01}$ in intrinsic coordinates of $S^{p} \times S^{q}$, we let $\tau$ be the azimuthal angle in $S^{p}$. That is, set

$$
\xi_{0}=\cos \tau, \quad 0 \leq \tau \leq \pi
$$

and complete $\tau$ to a set of spherical angular coordinates $\left(\tau, \tau_{1}, \ldots, \tau_{p-1}\right)$ on $S^{p}$. Likewise, set

$$
\xi_{1}=\cos \rho, \quad 0 \leq \rho \leq \pi,
$$

on $S^{q}$ and complete $\rho$ to a set of spherical angular coordinates $\left(\rho, \rho_{1}, \ldots, \rho_{q-1}\right)$ on $S^{q}$. Then

$$
\begin{aligned}
& L_{01}=\cos \rho \sin \tau \partial_{\tau}+\cos \tau \sin \rho \partial_{\rho}=: T, \\
& \omega_{01}=\cos \tau \cos \rho=: \varpi .
\end{aligned}
$$

Note that $\sin \tau \partial_{\tau}\left(\right.$ resp. $\left.\sin \rho \partial_{\rho}\right)$ is conformal on the Riemannian $S^{p}$ (resp. $S^{q}$ ) with the conformal factor $\cos \tau($ resp. $\cos \rho)$. 
Let $A=A_{2 r}$ be an intertwinor of order $2 r$ of the $(\mathfrak{g}, K)$ representation on functions over $S^{p} \times S^{q}$. That is, $A$ is a $K$-map satisfying the intertwining relation $[4,8]$

$$
A\left(\mathcal{L}_{X}+\left(\frac{n}{2}-r\right) \varpi\right)=\left(\mathcal{L}_{X}+\left(\frac{n}{2}+r\right) \varpi\right) A \quad \text { for all } X \in \mathfrak{g},
$$

where $\mathcal{L}_{X}$ is the Lie derivative.

Remark 3.1. If the intertwinor acts on tensors of $\left(\begin{array}{c}l \\ m\end{array}\right)$-type, then the Lie derivative should be changed to $\mathcal{L}_{X}+(l-m) \varpi$ in (3.2) [5, p. 347]. Note also that $\mathcal{L}_{X}=\nabla_{X}$ on functions.

The spectrum generating relation that converts (3.2) is given in the following lemma.

Lemma 3.1. Let $T$ and $\varpi$ be as in (3.1). Consider the Riemannian-Bochner Laplacian $\nabla^{*, \mathrm{R}} \nabla:=-g^{\alpha \beta} \nabla_{\alpha} \nabla_{\beta}=: N$, where $g=g_{S^{p}}+g_{S^{q}}$ is the standard Riemannian metric on $S^{p} \times S^{q}$. Then,

$$
[N, \varpi]=2\left(\nabla_{T}+\frac{n}{2} \varpi\right)
$$

on tensors of any type. Here $[\cdot, \cdot]$ is the usual operator commutator.

Proof. If $\varphi$ is any smooth section, then

$$
\begin{aligned}
{[N, \varpi] \varphi } & =(\triangle \varpi) \varphi-2 \iota(d \varpi) \nabla \varphi=\cos \rho \triangle_{S^{p}} \cos \tau+\cos \tau \triangle_{S^{q}} \cos \rho+2 \nabla_{T} \varphi \\
& =(p+q) \varpi+2 \nabla_{T} \varphi
\end{aligned}
$$

where $\iota$ is the interior multiplication and both $\triangle_{S^{p}}$ and $\triangle_{S^{q}}$ are Riemannian Laplacians.

Thus the intertwining relation (3.2) becomes

$$
A\left(\frac{1}{2}[N, \varpi]-r \varpi\right)=\left(\frac{1}{2}[N, \varpi]+r \varpi\right) A .
$$

Recall that the space of $j$-th order spherical harmonics on the Riemannian $S^{p}$ is the irreducible $\mathrm{SO}(p+1)$-module defined by

$$
E(j)=\left\{f \in C^{\infty}\left(S^{p}\right): \triangle_{S^{p}} f=j(j+p-1) f\right\}
$$

and the space $L^{2}\left(S^{p}\right)$ decomposes as

$$
L^{2}\left(S^{p}\right) \simeq \bigoplus_{j=0}^{\infty} E(j) .
$$

Let $F(k)$ be the space of $k$-th order spherical harmonics on the Riemannian $S^{q}$ and define

$$
\mathcal{V}(j, k):=E(j) \otimes F(k) .
$$

Note that we have a multiplicity free $K$-type decomposition into $K$-finite subspaces of the space of smooth functions on $S^{p} \times S^{q}$ :

$$
\bigoplus_{j, k=0}^{\infty} \mathcal{V}(j, k)
$$

and $K$ operator $A$ acts as a scalar multiplication on each $\mathcal{V}(j, k)$. 
On $S^{p}$, a proper conformal factor maps $E(j)$ to the direct sum $E(j+1) \oplus E(j-1)$. See [5] for details. Thus, a proper conformal factor on $S^{p} \times S^{q}$ maps a $K$-type $\mathcal{V}(j, k)$ to land in the direct sum of 4 types $\mathcal{V}\left(j^{\prime}, k^{\prime}\right)$ :

$$
\left(\begin{array}{ll}
\mathcal{V}(j-1, k+1) & \mathcal{V}(j+1, k+1) \\
\mathcal{V}(j-1, k-1) & \mathcal{V}(j+1, k-1)
\end{array}\right)
$$

Let $\alpha$ be the $K$-type $\mathcal{V}(j, k)$ and $\beta$ be any $K$-type appearing in (3.4). We apply the intertwining relation (3.3) to a section $\varphi$ in $\alpha$ :

$$
\begin{aligned}
A\left(\frac{1}{2}[N, \varpi]-r \varpi\right) \varphi & =\left(\frac{1}{2}[N, \varpi]+r \varpi\right) A \varphi \\
& \Leftrightarrow A\left(\frac{1}{2}\left(N(\varpi \varphi)-\varpi\left(N_{\alpha} \varphi\right)\right)-r \varpi \varphi\right)=\mu_{\alpha}\left(\frac{1}{2}\left(N(\varpi \varphi)-\varpi\left(N_{\alpha} \varphi\right)\right)+r \varpi \varphi\right),
\end{aligned}
$$

where $\mu_{\alpha}$ (resp. $N_{\alpha}$ ) is the eigenvalue of $A$ (resp. $N$ ) on the $K$-type $\alpha$. Note that $\varpi \varphi$ is a direct sum of the $K$-types appearing in (3.4). Let $\left.\operatorname{Proj}_{\beta} \varpi\right|_{a} \varphi$ be the projection of $\varpi \varphi$ onto the $K$-type $\beta$. The "compression", from the $K$-type $\alpha$ to the $K$-type $\beta$, of the above relation becomes $\left.\operatorname{Proj}_{\beta} \varpi\right|_{\alpha}$ times

$$
\left(\left.\frac{1}{2} N\right|_{\alpha} ^{\beta}+r\right) \mu_{\alpha}=\left(\left.\frac{1}{2} N\right|_{\alpha} ^{\beta}-r\right) \mu_{\beta},
$$

where $\mu_{\beta}$ (resp. $\left.N_{\beta}\right)$ is the eigenvalue of $A$ (resp. $N$ ) on the $K$-type $\beta$ and $\left.N\right|_{\alpha} ^{\beta}:=N_{\beta}-N_{\alpha}$. The underlined phrase above is a key point. We have achieved a factorization in which one factor is purely numerical (that appearing in (3.5)). "Canceling" the other factor, $\left.\operatorname{Proj}_{\beta} \varpi\right|_{\alpha}$, we get purely numerical recursions that are guaranteed to give intertwinors. If we wish to see the uniqueness of intertwinors this way, we need to establish the nontriviality of the $\left.\operatorname{Proj}_{\beta} \varpi\right|_{\alpha}$. In fact this nontriviality follows from Branson [5, Section 6].

To compute $\left.N\right|_{\alpha} ^{\beta}$, we need the following lemma.

Lemma 3.2. Let $\alpha=\mathcal{V}(j, k)$ and $\beta=\mathcal{V}\left(j^{\prime}, k^{\prime}\right)$.

$$
\left.N\right|_{\alpha} ^{\beta}=j^{\prime}\left(p-1+j^{\prime}\right)+k^{\prime}\left(q-1+k^{\prime}\right)-j(p-1+j)-k(q-1+k) .
$$

Proof. On the Riemannian $S^{p}, \nabla^{*} \nabla$ acts as (see [2] for details)

$$
j(p-1+j) \quad \text { on } E(j) \quad \text { and }\left.\quad N\right|_{\mathcal{V}(j, k)}=\left.\nabla^{*} \nabla\right|_{E(j)}+\left.\nabla^{*} \nabla\right|_{F(k)} .
$$

Let $J=j+\frac{p-1}{2}$ and $K=k+\frac{q-1}{2}$ for $j, k \in \mathbb{N}$. Then the transition quantities $\mu_{\beta} / \mu_{a}$ are

$$
\left(\begin{array}{cc}
\frac{-J+K+1+r}{-J+K+1-r} & \frac{J+K+1+r}{J+K+1-r} \\
\frac{-J-K+1+r}{-J-K+1-r} & \frac{J-K+1+r}{J-K+1-r}
\end{array}\right)
$$

relative to (3.4).

Note that $\mathcal{V}\left(f^{\prime}, j^{\prime}\right)$ can be reached from $\mathcal{V}(f, j)$ if and only if $\left|f^{\prime}-f\right|=\left|j^{\prime}-j\right|=1$. So $\mathcal{E}^{0}$, the direct sum of $\mathcal{V}(j, k)$ with $j+k$ even, is a $(\mathfrak{g}, K)$-invariant subspace of $\mathcal{E}\left(K, \lambda_{0}\right)$, as is the corresponding odd space $\mathcal{E}^{1}$. Choosing normalization $\mu_{\mathcal{V}(0,0)}=1\left(\right.$ resp. $\left.\mu_{\mathcal{V}(1,0)}=1\right)$ on $\mathcal{E}^{0}$ (resp. $\mathcal{E}^{1}$ ), we get 
Theorem 3.1. The unique spectral function $Z_{\varepsilon}(r ; f, j)$ on $\mathcal{E}^{\varepsilon}$ is, up to normalization,

$$
\frac{\Gamma\left(\frac{1}{2}(K+J+1+r)\right) \Gamma\left(\frac{1}{2}(K-J+1+r)\right) \Gamma\left(\frac{1}{2}\left(\varepsilon-\frac{p-q}{2}+1-r\right)\right) \Gamma\left(\frac{1}{2}\left(\varepsilon+\frac{p+q}{2}-r\right)\right)}{\Gamma\left(\frac{1}{2}(K+J+1-r)\right) \Gamma\left(\frac{1}{2}(K-J+1-r)\right) \Gamma\left(\frac{1}{2}\left(\varepsilon-\frac{p-q}{2}+1+r\right)\right) \Gamma\left(\frac{1}{2}\left(\varepsilon+\frac{p+q}{2}+r\right)\right)} .
$$

When $r$ is a positive integer, the spectral function provides conformally covariant differential operators. To see this, let

$$
B:=\sqrt{\triangle_{S^{p}}+\left(\frac{p-1}{2}\right)^{2}}, \quad C:=\sqrt{\triangle_{S^{q}}+\left(\frac{q-1}{2}\right)^{2}}
$$

so that $B$ and $C$ are nonnegative operators with

$$
\triangle_{S^{p}}=B^{2}-\left(\frac{p-1}{2}\right)^{2}, \quad \triangle_{S^{q}}=C^{2}-\left(\frac{q-1}{2}\right)^{2} .
$$

The eigenvalue list for $\triangle_{S^{p}}[2,11]$ is

$$
j(p-1+j), \quad j=0,1,2, \ldots,
$$

so the eigenvalue list for $B$ is

$$
j+\frac{p-1}{2}, \quad j=0,1,2, \ldots
$$

Similarly, the eigenvalue list for $C$ is

$$
k+\frac{q-1}{2}, \quad k=0,1,2, \ldots
$$

Note that for $r=1, Z_{\varepsilon}(1 ; f, j)$ is up to a constant

$$
\frac{1}{2}(K+J) \frac{1}{2}(K-J)
$$

Thus $Z_{\varepsilon}(1 ; f, j)$ agrees with a constant multiple of the conformal Laplacian on $S^{p} \times S^{q}$

$$
(C-B)(C+B)=\triangle_{S^{q}}-\triangle_{S^{p}}+\left(\frac{q-1}{2}\right)^{2}-\left(\frac{p-1}{2}\right)^{2}=\triangle_{S^{q}}-\triangle_{S^{p}}+\frac{n-2}{4(n-1)} \text { Scal },
$$

where Scal $=$ scalar curvature of $S^{p} \times S^{q}$.

In general, we have

Corollary $3.1([7])$. For a positive integer $r, Z_{\varepsilon}(r ; f, j)$ is a constant multiple of the differential operator

$$
(C+B-r+1) \cdots(C+B+r-1) \cdot(C-B-r+1) \cdots(C-B+r-1) .
$$

where the increments are by 2 units each time.

Remark 3.2. The same technique can be applied to the case of differential form bundles over $S^{p} \times S^{q}$. But here certain $K$-types occur with multiplicity 2 . On the multiplicity 1 types, exactly the same method yields the spectral function. 


\section{References}

[1] Branson T., Group representations arising from Lorentz conformal geometry, J. Funct. Anal. 74 (1987), 199-291.

[2] Branson T., Harmonic analysis in vector bundles associated to the rotation and spin groups, J. Funct. Anal. 106 (1992), 314-328.

[3] Branson T., Sharp inequalities, the functional determinant, and the complementary series, Trans. Amer. Math. Soc. 347 (1995), 3671-3742.

[4] Branson T., Nonlinear phenomena in the spectral theory of geometric linear differential operators, in Quantization, Nonlinear Partial Differential Equations, and Operator Algebra (Cambridge, MA, 1994), Proc. Sympos. Pure Math., Vol. 59, Amer. Math. Soc., Providence, RI, 1996, 27-65.

[5] Branson T., Stein-Weiss operators and ellipticity, J. Funct. Anal. 151 (1997), 334-383.

[6] Branson T.P., Hong D., Spectrum generating on twistor bundle, Arch. Math. (Brno) 42 (2006), suppl., 169-183, math.DG/0606524.

[7] Branson T., Hong D., Translation to bundle operators, SIGMA 3 (2007), 102, 14 pages, math.DG/0606552.

[8] Branson T., Ólafsson G., Ørsted B., Spectrum generating operators, and intertwining operators for representations induced from a maximal parabolic subgroups, J. Funct. Anal. 135 (1996), 163-205.

[9] Hong D., Eigenvalues of Dirac and Rarita-Schwinger operators, in Clifford Algebras (Cookeville, TN, 2002), Prog. Math. Phys., Vol. 34, Birkhäuser Boston, Boston, MA, 2004, 201-210.

[10] Hong D., Spectra of higher spin operators, Ph.D. Thesis, University of Iowa, 2004.

[11] Ikeda A., Taniguchi Y., Spectra and eigenforms of the Laplacian on $S^{n}$ and $P^{n}(\mathbb{C})$, Osaka J. Math. 15 (1978), 515-546.

[12] Kobayashi T., Ørsted B., Analysis on the minimal representation of $O(p, q)$. I. Realization via conformal geometry, Adv. Math. 180 (2003), 486-512, math.RT/0111083. 